\title{
Energy-momentum tensor and metric near the Schwarzschild sphere
}

\author{
Valentina Baccetti, ${ }^{1,2}$ Robert B. Mann, ${ }^{3,4}$ Sebastian Murk, ${ }^{1}$ and Daniel R. Terno ${ }^{1}$ \\ ${ }^{1}$ Department of Physics and Astronomy, Macquarie University, Sydney NSW 2109, Australia \\ ${ }^{2}$ School of Science, RMIT University, Melbourne, VIC 3000, Australia \\ ${ }^{3}$ Department of Physics and Astronomy, University of Waterloo, Waterloo, Ontario N2L 3G1, Canada \\ ${ }^{4}$ Perimeter Institute for Theoretical Physics, Waterloo, Ontario N2L 6B9, Canada
}

\begin{abstract}
Regularity of the horizon radius $r_{g}$ of a collapsing body constrains a limiting form of a spherically symmetric energy-momentum tensor near it. Its nonzero limit belongs to one of four classes that are distinguished only by two signs. As a result, close to $r_{g}$ the geometry can always be described by either an ingoing or outgoing Vaidya metric with increasing or decreasing mass. If according to a distant outside observer the trapped regions form in finite time, then the Einstein equations imply violation of the null energy condition. In this case the horizon radius and its rate of change determine the metric in its vicinity, and the hypersurface $r=r_{g}(t)$ is timelike during both the expansion and contraction of the trapped region. We present the implications of these results for the firewall paradox and discuss arguments that the required violation of the null energy condition is incompatible with the standard analysis of black hole evaporation.
\end{abstract}

\section{INTRODUCTION}

Astrophysical black holes (ABH) [1-3] - massive compact dark objects - are commonly found in the observable universe. Current data are consistent with having the Schwarzschild or Kerr-Newman solutions of classical general relativity (GR) as asymptotic final states of the collapse [2-4]. Nevertheless, the question of if, how and when ABHs develop any of the horizons and/or singularities that are predicted by GR [1-5] is still open [2, 3].

Quantum effects [1, 4, 6-12] add additional complexity. Event horizons may become optional $[4,10,13]$, and the notion of a black hole (BH) is then tied with one of the locally or quasilocally defined surfaces, such as an apparent horizon $[14,15]$. Hawking radiation accompanies the formation and evolution of black holes [16]. Its explicit form was originally obtained on the background of eternal black holes that are the vacuum solutions of GR $[1,7]$. Later it was shown that this phenomenon does not require formation of an event or even of an apparent horizon $[4,13,17]$. If the radiation is not terminated when the collapsing object reaches some macroscopic scale or becomes a Planck-scale remnant [12], the picture of an $\mathrm{ABH}$ as being in a permanent state of asymptotic approach to horizon formation becomes untenable as the object itself disappears in a finite time. Hawking radiation violates all energy conditions and thus provides additional possibilities both for having singularity-free objects with or without trapped regions, as well as naked singularities [3, 12].

Given that there are several related horizon definitions that are based on bounding the region of negative expansion of the outgoing null geodesics emanating from a spacelike compact two-dimensional surface with spherical topology, the definition of a physical BH (and thus the question of their very existence) is somewhat fuzzy. Hence we use the term "black hole" to designate any massive dense object that contains a trapped spacetime region, regardless of the presence of a suitably defined event horizon. They are observationally relevant physical objects (and not just useful mathematical idealizations) if formed at the finite time of a distant observer.

Our starting point is that such a BH exists; i.e., a trapped region is formed in a finite time $t_{\mathrm{S}}$ of Bob. We do not assume that this process is accompanied by Hawking radiation. If quantum effects lead to BH evaporation, our only assumption is that the evaporation time $t_{\mathrm{E}}>t_{\mathrm{S}}$. Our goal is to explore the implications of a compact object actually being a $\mathrm{BH}$.

We assume validity of semiclassical gravity $[17,18]$ and describe dynamics via the Einstein equations where the standard curvature terms are equated to the expectation value of the renormalized stress-energy tensor. It represents the entire matter content of the model: both the collapsing matter and the created excitations of the quantum fields are included. This cumulative representation allows a self-consistent study of the dynamics without having recourse to the usual iterative calculations of the backreaction [9].

We shall restrict our considerations to spherical symmetry, set $\hbar=c=G=1$ and use the -+++ signature.

\section{TENSORS NEAR THE SCHWARZSCHILD SPHERE}

Here we demonstrate how regularity of the apparent horizon constrains the metric in its vicinity. Moreover, if it is formed in a finite time of a distant observer, the family of allowed metrics is essentially unique.

\section{A. General considerations}

The most general spherically symmetric metric in $(z, r)$ coordinates, where $z$ is either the Schwarzschild time $t$ or the advanced or retarded null coordinate $u_{ \pm}$, is

$$
\begin{aligned}
d s^{2} & =-e^{2 h(t, r)} f(t, r) d t^{2}+f(t, r)^{-1} d r^{2}+r^{2} d \Omega \\
& =-e^{2 h_{ \pm}\left(u_{ \pm}, r\right)} f_{ \pm}\left(u_{ \pm}, r\right) d u_{ \pm}^{2} \pm 2 e^{h_{ \pm}\left(u_{ \pm}, r\right)} d u_{ \pm} d r+r^{2} d \Omega
\end{aligned}
$$

The function $f$ is coordinate independent $[19,20]$, i.e., $f(t, r)=f_{ \pm}\left(u_{ \pm}(t, r), r\right)$, and we can decompose it as

$$
f=1-C(t, r) / r=1-C_{ \pm}\left(u_{ \pm}, r\right) / r .
$$


The functions $h$ and $h_{ \pm}$play the role of integrating factors [20] that turn, e.g., the expression

$$
d t=e^{-h}\left(e^{h_{ \pm}} d u_{ \pm} \mp f^{-1} d r\right),
$$

into an exact differential, provided that the coordinate transformation exists.

In an asymptotically flat spacetime $h \rightarrow 0$ and $f \rightarrow 1$ as $r \rightarrow \infty$, and $t$ is the physical time of a stationary Bob at spacelike infinity. In the Schwarzschild spacetime $C=2 M$, and the coordinates $u_{ \pm}$are the retarded $u_{-} \equiv u:=t-r_{*}$ and advanced $u_{+} \equiv v:=t+r_{*}$ Eddington-Finkelstein coordinates, where $r_{*}$ is the tortoise coordinate.

On the other hand, having $h \rightarrow 0$ and $f \rightarrow 1-r^{2} / R_{H}$, where $R_{H}=1 / H_{\Lambda}$ is the Hubble radius, asymptotically describes a flat de Sitter space or a closed de Sitter space in static coordinates [21]. An explicit example of a gravitationally bound system in an expanding homogeneous and isotropic universe is furnished by the McVittie metrics [22], where late-time exponential expansion results in a de SitterSchwarzschild BH.

Regions of nonpositive expansion of outward pointing future-directed radial null geodesics (in asymptotically flat spacetimes) exist only if the equation $f(z, r)=0$ has a root $[15,20]$. This root (or, if there are several, the largest one) is the Schwarzschild horizon radius $r_{g}(z)$. Our first assumption is the existence of $r_{g}(t)$ for some period of time beginning at $t_{\mathrm{S}}<\infty$. If the spacetime is not asymptotically flat, we assume the separation of scales. In the de Sitter space example above, having stationary Bob's radial coordinate satisfy $r_{g} \ll r \ll R_{H}$, i.e., positioning him far outside the horizon radius and well inside the Hubble radius, puts him into an effectively flat region where $t$ is the physical time.

Regularity of the hypersurface $r=r_{g}$ is the standard assumption in the semiclassical theory $[1,2,8]$. Ricci and Kretschmann scalars have been used in investigations of $\mathrm{BH}$ evaporation without an event horizon [23]. We choose two scalars that are expressed directly in terms of the energymomentum tensor $T_{\mu \nu}$, namely, its trace $T:=T_{\mu}^{\mu} \equiv$ $R / 8 \pi$, where $R$ is the Ricci curvature scalar, and the square $\mathfrak{T}:=T^{\mu \nu} T_{\mu \nu}$. Our second assumption is that these scalars may have arbitrary but finite values, $\left|T\left(z, r_{g}\right)\right|<\infty$ and $\left|\mathfrak{T}\left(z, r_{g}\right)\right|<\infty$.

Finite curvature does not imply that the functions $h$ and $C$ in a particular coordinate system are finite. However, from the definition of $r_{g}$ it follows that

$$
C(t, r)=r_{g}(t)+W(t, x), \quad W(t, 0)=0,
$$

where $x:=r-r_{g}(t)$ and $W(t, x)<x$ for $x>0$. Derivatives of $W$ can diverge on the approach to $x=0$, but only moderately so as to ensure its continuity.

The Einstein equations that determine the functions $h$ and $C$ are

$$
\begin{aligned}
G_{t t} & =\frac{e^{2 h}(r-C) \partial_{r} C}{r^{3}}=8 \pi T_{t t}, \\
G_{t}^{r} & =\frac{\partial_{t} C}{r^{2}}=8 \pi T_{t}^{r} \\
G^{r r} & =\frac{(r-C)\left(-\partial_{r} C+2(r-C) \partial_{r} h\right)}{r^{3}}=8 \pi T^{r r} .
\end{aligned}
$$

This is the simplest form of the equations. It provides a natural choice of the independent components of the energymomentum tensor. The metric of Eq. (1) entails $T_{\theta}^{\theta} \equiv T_{\phi}^{\phi}$. Then the trace and the square scalars of the energy-momentum tensor are

$$
\begin{aligned}
& T=-e^{-2 h} T_{t t} / f+T^{r r} / f+2 T_{\theta}^{\theta}, \\
& \mathfrak{T}=-2\left(\frac{e^{-h} T_{t}^{r}}{f}\right)^{2}+\left(\frac{e^{-2 h} T_{t t}}{f}\right)^{2}+\left(\frac{T^{r r}}{f}\right)^{2}+2\left(T_{\theta}^{\theta}\right)^{2} .
\end{aligned}
$$

For future convenience we introduce $\tau_{t}:=e^{-2 h} T_{t t}, \tau^{r}:=$ $T^{r r}$ and $\tau_{t}^{r}:=e^{-h} T_{t}^{r}$.

Regularity of the invariants $T$ and $\mathfrak{T}$ holds independently of the left-hand side of the Einstein equations, which may involve quantum corrections [6]. Without any additional information we have to assume that the component $T_{\theta}^{\theta}$ is finite at $r_{g}$. If the dynamics is described by the Einstein equations, this intuitive property follows from the consistency of Eqs. (6)-(8) (see Appendix A 1).

The regularity of $T$ and $\mathfrak{T}$ at the Schwarzschild sphere implies that either the three limits of $\tau_{t}, \tau^{r}$ and $\tau_{t}^{r}$ are jointly zero as $r \rightarrow r_{g}$, approaching it at least as fast as $f(t, r)$, or the divergences in Eqs. (9) and (10) cancel out, so

$$
\lim _{r \rightarrow r_{g}} \tau^{r}=\lim _{r \rightarrow r_{g}} \tau_{t}=\Xi(t), \quad \lim _{r \rightarrow r_{g}} \tau_{t}^{r}= \pm \Xi(t),
$$

for some function $\Xi(t)$. Indeed, from Eq. (9) it follows that

$$
\lim _{r \rightarrow r_{g}} T=\lim _{r \rightarrow r_{g}}\left(-\tau_{t}+\tau^{r}\right) / f+2 T_{\theta}^{\theta}
$$

is finite, and thus $\tau_{t} \rightarrow \tau^{r}$ faster than $f \rightarrow 0$, and Eq. (10) implies $\left|\tau_{t}^{r}\right| \rightarrow\left|\tau_{r}\right|$. The form of the function $\Xi(t)$ is not constrained by regularity considerations.

We consider in detail the case $\Xi(t) \neq 0$, as it is more general and existing explicit calculations of $\left\langle\hat{T}_{\mu \nu}\right\rangle$ indicate that it is present $[1,7,24]$. The case of all three limits of $\tau_{t}, \tau^{r}$ and $\tau_{r}^{t}$ being $\Xi=:-\Upsilon^{2}$ is supported by the explicit calculations of the renormalized energy-momentum tensor for Unruh vacuum on the background of an eternal black hole (in that case $h \equiv 0$ ) [24], where the components $T^{r r}, T_{t t}$ and $T_{r}^{t}$ were shown to approach the same limit as $r \rightarrow r_{g}$. Appendix A 3 provides the details, as well as the analysis of the case $\Xi=0$ that leads to the same qualitative conclusions. We also consider here only the case of $T_{\mu \nu}$ that does not contain additional (milder) singular terms, as their presence does not change the leading terms in the solutions below. Close to $r_{g}$ Eq. (6) becomes

$$
\partial_{x} W \approx \frac{8 \pi \Xi r_{g}^{3}}{x-W} .
$$

Due to the singularity at $x=0$ Eq. (13) with the initial condition $W(t, 0)=0$ has two real solutions in terms of Lambert functions [25]. Both require $\Xi=-\Upsilon^{2}<0$ for some $\Upsilon(t)>0$. We will see below that this implies violation of the null energy condition (NEC) $[5,26]$. This is consistent with the well-known result that a trapped surface cannot be 
"visible" from future null infinity $\mathcal{I}^{+}$unless a weak energy condition is violated $[1,5]$. However, here it is a local result that is valid even if $\mathcal{I}^{+}$is not defined.

The auxiliary condition $W(t, x)<x$ selects the solution in terms of the Lambert function $W_{-1}$. Since the equation is approximate, we should use only the leading part of the solution that can also be obtained by direct evaluation of the series. It is

$$
W=-4 \Upsilon \sqrt{\pi r_{g}^{3} x}+\frac{1}{3} x \ldots,
$$

and it coincides with the leading part of the solution of the exact Einstein equation (6). In terms of $\alpha^{2}:=16 \pi \Upsilon^{2} r_{g}^{3}$, the mass function becomes

$$
C=\frac{2}{3} r_{g}(t)-\alpha(t) \sqrt{r-r_{g}(t)}+\frac{1}{3} r \ldots
$$

Substitution into Eq. (8) leads to

$$
\partial_{x} h \approx-\frac{8 \pi \Upsilon^{2} r_{g}^{3}}{(x-W)^{2}} \approx-\frac{\alpha^{2}}{2(\alpha \sqrt{x}+2 x / 3)^{2}} .
$$

Its exact solution is

$h=h_{0}(t)-\ln \frac{\sqrt{x}}{3 \alpha+2 \sqrt{x}}-\frac{3 \alpha}{3 \alpha+2 \sqrt{x}}=: h_{0}(t)+h_{1}(t, x)$.

The function of time $h_{0}(t)$ is not determined by the Einstein equations. Its choice determines the choice of the time variable. Higher-order terms depend on the exact form of $\tau_{t}$ and $\tau^{r}$. Hence

$$
h=-\ln \frac{\sqrt{x}}{\xi_{0}(t)}+\frac{4}{3 \alpha} \sqrt{x}+\mathcal{O}(x),
$$

where the function $\xi_{0}(t)$ is determined by the choice of the time variable. For example, expansion of Eq. (17) leads to $\xi_{0}=3 \alpha e^{h_{0}-1}$.

The flux sign is determined by the sign of $\partial_{t} C(t, r)$. Since (7) has to be consistent with (15), we match the singular part of $\partial_{t} C$ that is obtained from Eq. (7),

$$
\frac{\partial_{t} C}{r^{2}}=8 \pi T_{t}^{r} \approx \pm 8 \pi \Upsilon^{2} e^{h}= \pm \frac{8 \pi \Upsilon^{2} \xi_{0}}{\sqrt{r-r_{g}}}+\ldots
$$

with the singular part of

$$
\partial_{t} C=\dot{r}_{g}+\partial_{t} W=\frac{2 \Upsilon \sqrt{\pi r_{g}^{3}}}{\sqrt{r-r_{g}}} \dot{r}_{g}+\ldots,
$$

where the limit of the omitted terms is zero. As a result

$$
\dot{r}_{g} / \xi_{0}= \pm 4 \sqrt{\pi} \Upsilon \sqrt{r_{g}}= \pm \alpha / r_{g} .
$$

A direct calculation confirms that the scalars $R=R_{\mu}^{\mu}$ and $\mathfrak{R}=R^{\mu \nu} R_{\mu \nu}$ are indeed finite, as is the Kretschmann scalar $K=R^{\mu \nu \lambda \rho} R_{\mu \nu \lambda \rho}$. For example, the Ricci scalar can be expanded as

$$
R=\varrho_{3} x^{-3 / 2}+\varrho_{2} x^{-1}+\varrho_{1} x^{-1 / 2}+\mathcal{O}(x),
$$

where the coefficients of the divergent terms are

$$
\varrho_{3}=\left(\dot{r}_{g}^{2}-16 \pi \xi_{0}^{2} r_{g} \Upsilon^{2}\right) \rho_{3},
$$

and

$$
\begin{aligned}
\varrho_{2} & =\left(\dot{r}_{g}^{2}-16 \pi \xi_{0}^{2} r_{g} \Upsilon^{2}\right) \rho_{2}, \\
\varrho_{1} & =\rho_{1}\left(-32 \pi \xi_{0}^{3} r_{g} \Upsilon^{2}-18 \pi \dot{\xi}_{0} \dot{r}_{g} r_{g}^{3} \Upsilon^{2}\right. \\
& \left.+\xi_{0}\left[\left(2-9 \pi r_{g}^{2} \Upsilon^{2}\right) \dot{r}_{g}^{2}-18 \pi r_{g}^{3} \Upsilon\left(\dot{r}_{g} \dot{\Upsilon}-\Upsilon \ddot{r}_{g}\right)\right]\right),
\end{aligned}
$$

with the explicit form of the functions $\rho_{1,2,3}$ given in Appendix A 2, Eq. (A12).

The coefficients of $\varrho_{3}$ and $\varrho_{2}$ are identically zero due to Eq. (21), and the coefficient $\varrho_{1}$ is identically zero due to Eq. (21) and its derivative that relates $\dot{\Upsilon}$ and $\ddot{r}$.

At a fixed time qualitative changes in both the $h$ and $C$ functions occur at $x \sim \alpha^{2}=\dot{r}_{g}^{2} r_{g}^{2} / \xi_{0}^{2}$. For the distances $\alpha^{2} \lesssim x \ll r_{g}$ we have

$$
C(t, x) \approx r_{g}(t)+x / 3
$$

\section{B. Asymptotically flat spacetime}

A more explicit expression for $\xi_{0}$ can be obtained in an asymptotically flat spacetime. In this case the time $t$ is the physical time at spacelike infinity, hence $\lim _{x \rightarrow \infty} h(t, x)=0$, while

$$
h(t, x)=g_{0}(t)-\frac{1}{2} \ln x+\mathcal{O}(\sqrt{x}),
$$

for some function $g_{0}(t)$ for $x \rightarrow 0$ is satisfied by all spherically symmetric solutions with a finite-time formation of an apparent horizon.

We can decompose

$$
h(t, x)=h_{1}(t, x)+h_{2}(t, x),
$$

where

$$
\lim _{x \rightarrow \infty} h_{2}(t, x)=-\lim _{x \rightarrow \infty} h_{1}(t, x)=-\ln 2 .
$$

Series expansion as in Eq. (18) indicates that the higherorder terms in the energy-momentum tensor contribute only to the terms of the order $x$ or higher. On the other hand, this expansion does not set the limit of $h_{2}$ when $x \rightarrow 0$. If we can assume that it is zero, then

$$
g_{0}=\ln (3 \alpha)-1
$$

In a general asymptotically flat spacetime Eq. (7) becomes

$$
\frac{\partial_{t} C}{r^{2}}= \pm \frac{8 \pi \Upsilon^{2} e^{g_{0}}}{\sqrt{r-r_{g}}}+\ldots
$$

and if Eq. (30) applies,

$$
\dot{r}_{g}= \pm \frac{3 \alpha^{2}}{r_{g} e} .
$$




\section{Retarded and advanced coordinates}

Null coordinates $u_{ \pm}$allow for simpler metric expressions. In the case $\tau_{t}^{r}=-\Upsilon^{2}$ Eq. (4) leads to a particularly simple set of the Einstein equations in $\left(u_{+}, r\right)$ coordinates in the vicinity of $r_{g}=r_{+}$:

$$
\begin{aligned}
& \partial_{u_{+}} C_{+}=-8 \pi r_{+}^{2} e^{h_{+}} \Upsilon^{2}+\mathcal{O}\left(r-r_{+}\right), \\
& \partial_{r} C_{+}=\mathcal{O}\left(r-r_{+}\right), \quad \partial_{r} h_{+}=\mathcal{O}\left(r-r_{+}\right) .
\end{aligned}
$$

Their solution is regular,

$$
C_{+}=r_{+}\left(u_{+}\right)+\mathcal{O}\left(r-r_{+}\right)^{2}, \quad h_{+}=\mathcal{O}\left(r-r_{+}\right)^{2},
$$

where $d r_{+} / d u_{+}=-8 \pi r_{+}^{2} \Upsilon^{2}$, and the coefficients of the higher-order terms are determined by the full energymomentum tensor. Then close to the apparent horizon

$$
d s^{2}=-\left(1-r_{+} / r\right) d u_{+}^{2}+2 d u_{+} d r+r^{2} d \Omega .
$$

This form of the metric — ingoing Vaidya with decreasing mass - agrees with the near-horizon form of the metric of an evaporating $\mathrm{BH}[7]$.

Similarly, in the case $\tau_{t}^{r}=+\Upsilon^{2}$ the coordinates $\left(u_{-}, r\right)$ are particularly convenient. The Einstein equations become

$$
\begin{aligned}
& -\partial_{u_{-}} C_{-}=-8 \pi r_{+}^{2} e^{h_{-}} \Upsilon^{2}+\mathcal{O}\left(r-r_{-}\right), \\
& \partial_{r} C_{-}=\mathcal{O}\left(r-r_{-}\right), \quad \partial_{r} h_{-}=\mathcal{O}\left(r-r_{-}\right),
\end{aligned}
$$

resulting in the limiting form of the metric

$$
d s^{2}=-\left(1-r_{-} / r\right) d u_{-}^{2}-2 d u_{-} d r+r^{2} d \Omega,
$$

which is the outgoing Vaidya metric with increasing mass.

\section{NULL ENERGY CONDITION NEAR THE SCHWARZSCHILD SPHERE}

The limiting form of the $(t r)$ block of $T_{\mu \nu}$ is

$$
T_{b}^{a}=\left(\begin{array}{cc}
-\Xi / f & -s e^{-h} \Xi / f^{2} \\
s e^{h} \Xi & \Xi / f
\end{array}\right), \quad T_{\hat{a} \hat{b}}=\frac{\Xi}{f}\left(\begin{array}{ll}
1 & s \\
s & 1
\end{array}\right),
$$

where $s= \pm 1$ and the second expression is written in the orthonormal frame. This result is independent of the Einstein equations and remains valid even if higher-order curvature terms are added to their left-hand side.

For $\Xi=-\Upsilon^{2}$ and $s= \pm 1$ the NEC is violated; $T_{\hat{a} \hat{b}} k^{\hat{a}} k^{\hat{b}}<$ 0 for a radial null vector $k^{\hat{a}}=(1, s, 0,0)$. This is the case we have considered in Sec. II. Using the metric in $\left(u_{ \pm}, r\right)$ coordinates we see that the Schwarzschild sphere is the outer boundary of spherically symmetric marginally trapped surfaces.

Unlike models of collapse that satisfy energy conditions, the finite-time Schwarzschild sphere is timelike during both its growth and shrinking. Indeed, in the growth phase we parametrize it as a hypersurface

$$
\Phi(x)=r-r_{-}\left(u_{-}\right)=0 .
$$

Then the normal $n_{\mu} \propto \partial_{\mu} \Phi$ satisfies $n_{\mu} n^{\mu} \propto 2 d r_{-} / d u_{-}>0$, and thus the surface is timelike. The same can be observed by finding that $k^{2}<0$, where $k=\left(1, r_{-}^{\prime}, 0,0,\right)$ is the tangent vector to the hypersurface $r=r_{g}$. During evaporation $r_{+}^{\prime}<0$, the parametrization $\Phi(x)=r-r_{+}\left(u_{+}\right)=0$ leads to $\partial_{\mu} \Phi \partial^{\mu} \Phi=-2 d r_{+} / d u_{+}>0$.

When the energy density is given by $\Xi=\Upsilon^{2}>0$, the Schwarzschild sphere forms only "beyond the end of time." Taking $s=-1$, a formal transformation to $\left(u_{-}, r\right)$ coordinates gives

$$
\begin{aligned}
& \partial_{u_{-}} C_{-} \approx-8 \pi r_{-}^{2} e^{h_{-}} \Upsilon^{2}, \\
& \partial_{r} C_{-} \approx 0, \quad \partial_{r} h_{-} \approx 0 .
\end{aligned}
$$

Their solutions are

$$
C_{-}=r_{-}+\mathcal{O}\left(r-r_{-}\right)^{2}, \quad h_{-}=\mathcal{O}\left(r-r_{-}\right)^{2},
$$

with the higher-order terms determined by the full energymomentum tensor. The leading terms correspond to the pure outgoing Vaidya metric with $C_{-}^{\prime}<0$. Likewise, if $s=+1$, we obtain the ingoing Vaidya metric with $C_{+}^{\prime}>0$ up to leading order in $\left(r-r_{+}\right)$. The four cases of the limiting form of $T_{\mu \nu}$ are summarized in Table 1.

\begin{tabular}{c|c|c|cc}
\hline $\operatorname{sgn}\left(T_{t t}\right)$ & $\operatorname{sgn}\left(T_{t}^{r}\right)$ & $s$ & \multicolumn{2}{|c}{ Vaidya metric } \\
\hline \hline- & - & + & $\left(u_{+}, r\right)$ & $C^{\prime}\left(u_{+}\right)<0$ \\
\hline- & + & - & $\left(u_{-}, r\right)$ & $C^{\prime}\left(u_{-}\right)>0$ \\
\hline+ & - & - & $\left(u_{-}, r\right)$ & $C^{\prime}\left(u_{-}\right)<0$ \\
\hline+ & + & + & $\left(u_{+}, r\right)$ & $C^{\prime}\left(u_{+}\right)>0$ \\
\hline
\end{tabular}

TABLE I. Signs in the limiting form of $T_{\mu \nu}$. The Einstein equations have real solutions only in the first two cases.

In these coordinates the existence of an apparent horizon radius $r_{ \pm}$is compatible with positive energy density. However, since violation of the energy conditions is a coordinateindependent property, there is no transformation between $\left(u_{ \pm}, r\right)$ coordinates to $(t, r)$ coordinates when $\Xi>0$. Indeed, when such a transformation is constructed (Appendix $\mathrm{C}$ ), it results in complex-valued functions $t\left(u_{ \pm}, r\right)$.

If a trapped region appears at finite time $t_{\mathrm{S}}$ of $\mathrm{Bob}$, it can be maintained only if the total matter content outside it violates the NEC and thus all energy conditions. This must hold regardless of the presence or absence of the Hawking-like radiation that is observable by Bob. As a result, the question of the existence of a $\mathrm{BH}$ (that we take to be an $\mathrm{ABH}$ with a trapped region inside, with or without singularities) becomes the question of the possibility of maintaining the required violation of the energy conditions $[26,27]$ for a sufficient amount of time.

We now consider the implications of quantum energy inequalities (QEI) for our results. These inequalities generalize the classical positive energy conditions and arise because classical energy conditions do not hold pointwise in a quantum field theory [27]. They necessarily invoke time averages, as there are no explicit restrictions on spatial averages [28]. We assume that the lifetime of the evaporating $\mathrm{BH}$ is predicted by the usual analysis $[1,8]$, i.e., by $\Delta t_{\mathrm{E}} \propto M_{0}^{3}$, where $M_{0}$ is 
the gravitational mass at the appearance of the Schwarzschild sphere.

The rigorous answer can be obtained by adapting the inequalities bounding violation of the NEC [27, 29] to the relevant near-horizon metrics. There is a one-to-one relationship between a static observer near an event horizon of a Schwarzschild BH and a uniformly accelerated observer in Minkowski spacetime. We exploit its approximate applicability in our time-dependent scenario [30].

Reference [29] established a QEI for an observer moving with uniform acceleration $w^{\mu}$ on the trajectory $\gamma$ that is parametrized by the proper time $\tau$ in Minkowski spacetime (or a spacetime that can be mapped to Minkowski under a precise set of conditions). Then for any Hadamard state $\omega$ and a smooth function $\mathfrak{f}(\tau)$ of compact support, $\int_{-\infty}^{\infty} \mathfrak{f}^{2}(\tau) d \tau=1$, the negativity of the energy-momentum expectation value is bounded by

$$
\lim _{\tau_{0} \rightarrow \infty} \inf \frac{1}{\tau_{0}} \int_{\gamma} \mathfrak{f}\left(\tau / \tau_{0}\right)^{2}\left\langle\hat{T}_{\mu \nu}\right\rangle_{\omega} u^{\mu} u^{\nu} d \tau \geqslant-\frac{11 w^{4}}{480 \pi^{2}}
$$

We apply this bound to a stationary observer Eve in the vicinity of the Schwarzschild sphere of a slowly evaporating BH. In $\left(u_{+}, r\right)$ coordinates the metric is given by Eq. (36) and her four-velocity by $u_{\text {Eve }}^{\mu}=(1 / \sqrt{f}, 0,0,0)$. Thus Eve's energy density is

$$
\rho_{\mathrm{Eve}}=T_{\mu \nu} u_{\mathrm{Eve}}^{\mu} u_{\mathrm{Eve}}^{\nu} \approx-\frac{\Upsilon^{2}}{f} \approx \frac{C_{+}^{\prime}}{8 \pi C_{+} x} .
$$

It is useful to introduce the reduced variables $\chi:=x / C_{+}$and $\beta:=-C_{+}^{\prime} / C_{+}$. The near-horizon approximation is valid for $\chi \ll 1$, while the Planck scale corresponds to $x=\chi C_{+}=1$. For Eve at rest close to the Schwarzschild sphere, the fourth power of the four-acceleration satisfies

$$
w_{\mathrm{Eve}}^{4}=\frac{\left(\chi-\beta C_{+}(1+\chi)^{2}\right)^{4}}{16 C_{+}^{4} \chi^{6}(1+\chi)^{6}},
$$

where for a macroscopic $\mathrm{BH}$ we expect $\beta \ll 1$. This quantity diverges as $\chi \rightarrow 0$, but it sharply drops to zero and stays close to it in a narrow interval at the sub-Planckian value

$$
\xi_{0}=\beta C_{+},
$$

while $\rho_{\text {Eve }} \approx \beta /\left(8 \pi \xi_{0}\right)$. We expect Eq. (45) to be applicable if it is possible to have $\tau_{0}$ long enough, i.e., $w_{\mathrm{Eve}} \tau_{0} \gg 1$, which is still short enough on the evaporation scale, $\Delta u_{+} \ll$ $u_{+}^{\mathrm{E}}$. The proper time is obtained via $d \tau=\sqrt{f} d v$. Then (assuming that Eve is outside of the sub-Planckian region, i.e., $\left.\chi \gg \beta C_{+}\right)$

$$
\tau_{0} \approx \sqrt{\frac{x}{C_{+}}} \Delta u_{+}=\sqrt{\chi} \Delta u_{+} \gg 2 C_{+} \sqrt{\chi},
$$

where $C_{+}$is approximately constant during $\tau_{0}$, and the double bound is

$$
u_{+}^{\mathrm{E}} \gg \Delta u_{+} \gg C_{+},
$$

which can be satisfied for a macroscopic BH. The estimate does not change when $\chi \sim \beta C_{+}$. Approximating the sampling function $\mathfrak{f}^{2}$ by a box of width $\tau_{0}$, we approximate the left-hand side of Eq. (45) as $\rho_{\mathrm{E}}$.

Comparing it with $w_{\text {Eve }}^{4}$ we find that the continuous domain where the QEI is satisfied belongs to the sub-Planckian regime, while calculations that include backreaction of the Hawking radiation energy-momentum tensor on the geometry lead to Eq. (36) as the leading correction valid for distances that are comparable with $r_{g}$ [7]. Hence the above estimate can indicate either that the required negative energy density for having a Schwarzschild sphere at finite time $t_{\mathrm{S}}$ cannot be maintained, or that the Vaidya approximation of Eq. (36) to the near-horizon geometry is valid in a much narrower regime than previously thought. The latter may be another indication that the semiclassical approximation and its associated classical notions are modified already at the horizon or larger scales.

If we ignore this narrow sub-Planckian feature of $w_{\text {Eve }}^{4}$, we see that the QEI is satisfied at larger scales, but the extent of the possible NEC-violating region depends on the details of evaporation. This can be seen as follows. For a sufficiently slow evaporation $\left(\beta C^{2} \ll 1\right.$, which we expect to be true for macroscopic BHs), and for distances above the Planck scale $\chi \gtrsim 1 / C_{+}$, we can approximate $w_{\text {Eve }}^{4}$ by setting $\beta=0$. Then the QEI becomes

$$
\frac{11}{960 C_{+}^{4} \pi \chi(1+\chi)^{6}} \geqslant \beta .
$$

For $\beta C_{+}^{4} \lesssim 1$ it is satisfied at macroscopic scales, but with increasing (even still satisfying $\beta C_{+}^{3} \ll 1$ ) evaporation rates

$$
\chi<\frac{11}{960 \pi \beta C_{+}^{4}},
$$

again pushing the NEC-violating regime to the Planck scale.

\section{DISCUSSION}

We demonstrated that having a trapped region appear in finite time from the perspective of a distant observer not only leads to the violation of energy conditions but, together with the requirement of regularity of its boundary, determines the limiting form of the total energy-momentum tensor and the metric near the Schwarzschild sphere. This physically motivated classification of the energy-momentum tensor near $r_{g}$ and the explicit expression for the metric allows to resolve the controversies that surround the thin shell models of collapse [31].

If the singularity forms at some finite $t$ (as may happen even if some energy conditions are violated [11]), a version of the information loss problem may be posited $[4,8]$. The firewall paradox plays a prominent role among its numerous aspects. Our analysis clarifies what the "no drama at the horizon" postulate that is used in its derivation actually means. So long as near the Schwarzschild sphere the curvature remains finite, the resulting average energy density as perceived by the infalling Alice is negative, as can be seen from Eq. (40). Hence 
she does not see the vacuum, which is one of the elements in the chain of arguments at least in some versions of the firewall paradox [8]. Moreover, if the flux of energetic particles is desired to disentangle the early and late modes of emitted radiation, it has to not only avoid being a universal disentangler (forbidden by unitarity) [32], but also be weak enough so as to not destroy the horizon while being energetic enough for the erasure of information (at or above the Landauer bound) [33]. This is a highly nontrivial requirement. Indeed, such a burst may still be inefficient in destroying the correlations while causing the renormalized energy density $\left\langle\hat{T}_{00}\right\rangle$ to diverge to positive infinity in the energy pulse [34].

Several problems present themselves as the logical extension of this work: (i) existence of trapped regions at finite $t$ with axial symmetry; (ii) rigorous derivation of the QEI for the resulting geometries and study of their implications on $\mathrm{BH}$ existence; this will also include evaluation of the limiting value $g_{0}(t)$; (iii) investigation of the Schwarzschild radius, energy conditions and metric in alternative theories of gravity; (iv) finding possible observational differences with the black holes of GR, particularly via quasinormal modes.

\section{ACKNOWLEDGMENTS}

We thank Amos Ori and Paddy Padmanabhan for important critical comments and Mark Wardle for useful discussions on astrophysical black holes. The work of RBM was supported in part by the Natural Sciences and Engineering Research Council of Canada. SM is supported by the iMQRES scheme of Macquarie University.

\section{Appendix A: Metric near $r_{g}(t)$ and different limiting forms of the energy-momentum tensor}

\section{Regularity of $\mathbf{T}_{\theta}^{\theta}$}

If we assume that $T_{\theta}^{\theta}$ diverges at $r_{g}$, then the finiteness of the scalars $T$ and $\mathfrak{T}$ requires that it also has the asymptotic form $\Omega / f$. As a result the regularity conditions become

$$
-\Xi+\Psi+2 \Omega=0, \quad-2 \Sigma^{2}+\Xi^{2}+\Psi^{2}+2 \Omega^{2}=0,
$$

where

$$
\begin{aligned}
& \lim _{r \rightarrow r_{g}} \tau^{r}=\Psi(t), \\
& \lim _{r \rightarrow r_{g}} \tau_{t}=\Xi(t), \\
& \lim _{r \rightarrow r_{g}} \tau_{t}^{r}=\Sigma(t) .
\end{aligned}
$$

Taking $\Xi$ and $\Psi$ as independent variables we find

$$
\Omega=\frac{1}{2}(\Xi-\Psi), \quad \Sigma^{2}=\frac{1}{4}\left(3 \Xi^{2}+3 \Psi^{2}-2 \Xi \Psi\right) .
$$

The Einstein equation (6) does not change; its solution is

$$
C=r_{g}+W(t, x)=\frac{2}{3} r_{g}-4 \Upsilon_{\Xi} \sqrt{\pi r_{g}^{3}\left(r-r_{g}\right)}+\frac{1}{3} r \ldots,
$$

where $\Xi=-\Upsilon_{\Xi}^{2}$. However, Eq. (8) becomes

$$
\partial_{x} h=4 \pi \frac{\left(\Sigma-\Upsilon_{\Xi}^{2}\right)}{(x-W)^{2}}
$$

Its solution is

$$
h=h_{0}(t)+\frac{\Sigma-\Upsilon_{\Xi}^{2}}{4 \Upsilon_{\Xi}^{2}} \ln \left(r / r_{g}-1\right)+\ldots
$$

Hence, on the one hand,

$$
\partial_{t} C=\frac{2 \Upsilon_{\Xi} \sqrt{\pi r_{g}^{3}}}{\sqrt{r-r_{g}}} \dot{r}_{g}+\ldots
$$

and it can be matched with

$$
\frac{\partial_{t} C}{r^{2}}=8 \pi T_{t}^{r} \approx \pm 8 \pi \Sigma e^{h}
$$

only if $h \propto x^{-1 / 2}$, implying $\Psi=\Xi=-\Upsilon^{2}$. Hence $\Omega=0$ and $T_{\theta}^{\theta}$ is finite at the Schwarzschild sphere.

\section{The Ricci scalar}

The explicit form of the Ricci tensor is given by

$$
\begin{aligned}
R r^{2}= & -(4 r-C) \partial_{r} h-2 r(r-C)\left(\partial_{r} h\right)^{2} \\
& +\partial_{r} C\left(2+3 r \partial_{r} h\right)+r \partial_{r}^{2} C-2 r(r-C) \partial_{r}^{2} h \\
& +\frac{e^{-2 h} r^{3}}{(r-C)^{3}}\left(2\left(\partial_{t} C\right)^{2}-(r-C) \partial_{t} C \partial_{t} h\right. \\
& \left.+(r-C) \partial_{t}^{2} C\right) .
\end{aligned}
$$

Expansion in powers of $x=r-r_{g}$ results in the Laurent series

$$
\begin{gathered}
R=\varrho_{3} x^{-3 / 2}+\varrho_{2} x^{-1}+\varrho_{1} x^{-1 / 2}+\mathcal{O}(x) \\
=\frac{p_{3}}{8 \sqrt{\pi r_{g}} \xi_{0}^{2} \Upsilon} \frac{1}{x^{3 / 2}}+\frac{p_{2}}{16 \pi r_{g}^{2} \xi_{0}^{2} \Upsilon^{2}} \frac{1}{x} \\
\quad+\frac{p_{1}}{144 \pi^{3 / 2} \xi_{0}^{3} r_{g}^{7 / 2} \Upsilon^{3}} \frac{1}{\sqrt{x}}+\mathcal{O}\left(x^{0}\right),
\end{gathered}
$$

in $\sqrt{x}$, with

$$
\begin{aligned}
p_{3} & =\left(\dot{r}_{g}^{2}-16 \pi \xi_{0}^{2} r_{g} \Upsilon^{2}\right), \\
p_{2} & =\dot{r}_{g}^{2}-16 \pi \xi_{0}^{2} r_{g} \Upsilon^{2} \\
p_{1} & =-32 \pi \xi_{0}^{3} r_{g} \Upsilon^{2}-18 \pi \dot{\xi}_{0} \dot{r}_{g} r_{g}^{3} \Upsilon^{2} \\
& +\xi_{0}\left[\left(2-9 \pi r_{g}^{2} \Upsilon^{2}\right) \dot{r}_{g}^{2}-18 \pi r_{g}^{3} \Upsilon\left(\dot{r}_{g} \dot{\Upsilon}-\Upsilon \ddot{r}_{g}\right)\right] .
\end{aligned}
$$

where the denominators are the functions $\rho_{i}$ that were defined in Sec. II.

\section{The case $\Xi=0$}

Here we discuss the case of the limits in Eq. (11) being zero. Continuity of $T$ and $\mathfrak{T}$ does not impose any additional 
restrictions on their behavior; we summarize this situation as

$$
\begin{aligned}
& \lim _{r \rightarrow r_{g}} \frac{e^{-2 h} T_{t t}}{f^{k}}=A(t), \\
& \lim _{r \rightarrow r_{g}} \frac{T^{r r}}{f^{l}}=B(t), \\
& \lim _{r \rightarrow r_{g}} \frac{e^{-h} T_{t}^{r}}{f^{m}}=L(t),
\end{aligned}
$$

for some $k, l, m \geq 1$, where a priori there is no relationship between the functions $A, B$ and $L$.

Consider first the simplest case $k=1$. For regular functions we have to take into account the next term in the expansion of the $\tau_{t}$ and other components in $r-r_{g}$. Hence close to $r_{g}$ Eq. (6) implies

$$
\partial_{r} C \approx 8 \pi\left(A+A_{1}\left(r-r_{g}\right)\right) r_{g}^{2},
$$

where $A_{1}:=3 r_{g}^{2} A$. Its solution is

$$
C=r_{g}+8 \pi A r_{g}^{2}\left(r-r_{g}\right)+\left(4 \pi A_{1} r_{g}^{2}\right)\left(r-r_{g}\right)^{2}+\ldots,
$$

where the requirement $W<x=r-r_{g}$ for $x>0$ leads to the inequality

$$
8 \pi A r_{g}^{2} \leq 1
$$

If the inequality is strict, i.e., $8 \pi A r_{g}^{2}<1$, then the function $h$ either diverges at least logarithmically due to

$$
\partial_{r} h \approx \frac{4 \pi(A+B) r^{2}}{r-C} \approx \frac{4 \pi(A+B) r_{0}^{2}}{\left(1-8 \pi A r_{g}^{2}\right)\left(r-r_{g}\right)},
$$

or is regular if $A=-B$. Matching the two expressions for $\partial_{t} C$ at $r=r_{g}$ requires

$$
\partial_{t} C=\left(1-8 \pi A r_{g}^{2}\right) \dot{r}_{g}=r_{g}^{2} L \lim _{r \rightarrow r_{g}} e^{h} f^{m} .
$$

This identity can be satisfied only if $h$ diverges logarithmically with a coefficient $\mu=-1$ while $m=1$. Hence (in a gauge where $\xi_{0}=\sqrt{r_{g}}$ )

$$
\frac{4 \pi(A+B) r_{g}^{2}}{\left(1-8 \pi A r_{g}^{2}\right)}=-1, \quad \dot{r}_{g}=\frac{L r_{g}^{2}}{\left(1-8 \pi A r_{g}^{2}\right)}
$$

We find that $R$ and $\Re$ are finite at $r_{g}$ if an additional condition

$$
\left(1-8 \pi A r_{g}^{2}\right)^{2}=\dot{r}_{g}^{2}
$$

is satisfied, relating $A$ and $L$.

On the other hand, if $8 \pi A r_{g}^{2}=1$, then

$$
C=r-\beta^{2} x^{2},
$$

where $\beta^{2}=-\left(8 \pi A r_{g}+4 \pi A_{1} r_{g}^{2}\right)$. In this case $\partial_{t} C_{0}=0$. The function $h$ either diverges at least as $1 / x$ due to

$$
\partial_{r} h \approx \frac{4 \pi(A+B) r^{2}}{r-C_{0}} \approx-\frac{4 \pi(A+B) r_{0}^{2}}{\beta^{2} x^{2}},
$$

or is regular if $A=-B$. Since $\exp (1 / x)$ diverges faster than any inverse power of $x$, the identity

$$
0=\partial_{t} C=r_{g}^{2} L \lim _{r \rightarrow r_{g}} e^{h} f^{m}
$$

can be satisfied only if $A=-B$ (thus getting a finite $h$ ) and $m \geq 1$. Nevertheless, the Ricci scalar diverges, unless both $\partial_{t} C_{0}$ and $\partial_{t}^{2} C_{0}$ are zero. Hence this case is excluded as it is not compatible with the evolution of the Schwarzschild radius. As a result, the simplest case of $\Xi=0$ is given by $k=l=$ $m=1$, and

$$
\begin{aligned}
& B=\frac{-1+4 A \pi r_{g}^{2}}{4 \pi r_{g}^{2}}=A-\frac{1}{4 \pi r_{g}^{2}}, \\
& L= \pm \frac{\left(1-8 \pi A r_{g}^{2}\right)^{2}}{r_{g}^{2}}, \quad 1>8 \pi A r_{g}^{2}
\end{aligned}
$$

In this case the $(t r)$ block of the energy-momentum tensor in the orthonormal frame is

$$
T_{\hat{a} \hat{b}}=\left(\begin{array}{ll}
A & L \\
L & B
\end{array}\right),
$$

where $B(A)$ and $L(A)$ and the bound on $A$ are given in Eq. (A30). Using these relations we find that both for positive and negative $A$ the NEC is violated $\left(T_{\hat{a} \hat{b}} k^{\hat{a}} k^{\hat{b}}<0\right)$ with the incoming radial null geodesic $k^{\hat{a}}=(1,-1,0,0)$.

Appendix B: $\left(u_{ \pm}, r\right)$ coordinates and the Einstein equations: $\Xi<0$

In this section we present the case of negative energy density when the partial differential equation (PDE) for the integrating factor $h_{ \pm}$has a real-valued solution, meaning that we are simply discussing the same physical situation in different coordinate systems. We denote the energy-momentum tensor in the two coordinate systems $\left(u_{ \pm}, r\right)$ as $\Theta_{\mu \nu}^{ \pm}$, respectively. To reduce clutter we restore $u=u_{-}$and $v=u_{+}$.

\section{1. $(u, r)$ coordinates}

First we analyze the retarded null coordinate. Note that the Schwarzschild radius satisfies $r_{-}(u)=r_{g}\left(t\left(u, r_{-}(u)\right)\right)$, so the numerical values of limits $\Upsilon$ are the same. Recall

$$
d t=e^{-h}\left(e^{h_{-}} d u+d r / f\right) .
$$

Hence the relevant components of the energy-momentum tensor for the two signs of the flux $\tau_{t}^{r}=-\Upsilon^{2}\left(\tau_{t}^{r}=+\Upsilon^{2}\right)$ are

$$
\begin{aligned}
& \Theta_{u u}^{-}=\left(\frac{\partial t}{\partial u}\right)^{2} T_{t t} \rightarrow-e^{2 h_{-}} \Upsilon^{2} \quad\left(-e^{2 h_{-}} \Upsilon^{2}\right), \\
& \Theta_{u r}^{-}=\frac{\partial t}{\partial u} \frac{\partial t}{\partial r} T_{t t}+\frac{\partial t}{\partial u} T_{t r} \rightarrow-\frac{2}{f} e^{h_{-}} \Upsilon^{2} \quad(0), \\
& \Theta_{r r}^{-}=T_{r r}+\left(\frac{\partial t}{\partial r}\right)^{2} T_{t t}+2 \frac{\partial t}{\partial r} T_{t r} \rightarrow-\frac{4}{f^{2}} \Upsilon^{2}
\end{aligned}
$$


In both cases the left-hand sides of the Einstein equations are

$$
\begin{aligned}
G_{u u} & =e^{2 h_{-}} \frac{\partial_{r} C_{-}}{r^{2}}\left(1-\frac{C_{-}}{r}\right)-e^{h_{-}} \frac{\partial_{u} C_{-}}{r^{2}}, \\
G_{u r} & =e^{h_{-}} \frac{\partial_{r} C_{-}}{r^{2}} \\
G_{r r} & =\frac{2 \partial_{r} h_{-}(u, r)}{r} .
\end{aligned}
$$

Close to the Schwarzschild sphere we get (for $\tau_{t}^{r}=-\Upsilon^{2}$, contraction of the apparent horizon)

$$
\begin{aligned}
& \partial_{u} C_{-}=-8 \pi r_{-}^{2} e^{h_{-}} \Upsilon^{2}, \\
& \partial_{r} C_{-}=-16 \pi r_{-}^{2} \Upsilon^{2} / f, \\
& \partial_{r} h_{-}=-16 \pi r_{-} \Upsilon^{2} / f^{2} .
\end{aligned}
$$

The second and the third equation give

$$
C_{-}(u, r)=\frac{2}{3} r_{-}(u)-\sqrt{2} a\left(r-r_{-}\right)^{1 / 2}+\frac{1}{3} r \ldots,
$$

and

$$
h_{-}=-\frac{1}{2} \ln \left(r / r_{-}-1\right)+\frac{2 \sqrt{2}}{3} \frac{\sqrt{x}}{a},
$$

where

$$
a:=4 \sqrt{\pi} \Upsilon r_{-}^{3 / 2} .
$$

Similar to the analysis in $(t, r)$ coordinates we have the identity between the two ways to calculate $\partial C_{-}\left(u, r_{-}\right)$. On the one hand the Einstein equation implies

$$
\lim _{r \rightarrow r_{-}} \partial_{u} C_{-}(u, r)=-\frac{a^{2}}{2 \sqrt{r_{-}\left(r-r_{-}\right)}},
$$

and on the other hand

$$
\lim _{r \rightarrow r_{-}} \partial_{u} C_{-}(u, r)=\frac{a d r_{-} / d u}{\sqrt{2\left(r-r_{-}\right)}} .
$$

Hence

$$
\frac{d r_{-}}{d u}=-\frac{a}{\sqrt{2 r_{-}}}
$$

\section{2. $(v, r)$ coordinates}

The relevant components of the energy-momentum tensor for the two signs of the flux $\tau_{t}^{r}=-\Upsilon^{2}\left(\tau_{t}^{r}=+\Upsilon^{2}\right)$ are

$$
\begin{aligned}
& \Theta_{v v}^{+}=\left(\frac{\partial t}{\partial v}\right)^{2} T_{t t} \rightarrow-e^{2 h_{+}} \Upsilon^{2}\left(-e^{2 h_{+}} \Upsilon^{2}\right), \\
& \Theta_{v r}^{+}=\frac{\partial t}{\partial v} \frac{\partial t}{\partial r} T_{t t}+\frac{\partial t}{\partial v} T_{t r} \rightarrow 0 \quad\left(\frac{2}{f} e^{h_{+}} \Upsilon^{2}\right), \\
& \Theta_{r r}^{+}=T_{r r}+\left(\frac{\partial t}{\partial r}\right)^{2} T_{t t}+2 \frac{\partial t}{\partial r} T_{t r} \rightarrow 0 \quad\left(\frac{4}{f^{2}} \Upsilon^{2}\right) .
\end{aligned}
$$

The Einstein equations are

$$
\begin{aligned}
G_{v v} & =e^{2 h_{2}} \frac{\partial_{r} C_{+}}{r^{2}}\left(1-\frac{C_{+}}{r}\right)+e^{h_{+}} \frac{\partial_{v} C_{+}}{r^{2}}, \\
G_{v r} & =-e^{h_{+}} \frac{\partial_{r} C_{+}}{r^{2}} \\
G_{r r} & =\frac{2 \partial_{r} h_{+}(v, r)}{r} .
\end{aligned}
$$

Appendix C: $\left(u_{ \pm}, r\right)$ coordinates and the Einstein equations: $\Xi>0$

The case $\Xi=\Upsilon^{2}$ with negative $\tau_{t}^{r}$ is most conveniently described using retarded null coordinates

$$
\begin{aligned}
\partial_{u} C_{-} & =-8 \pi r_{-}^{2} \Upsilon^{2}, \\
\partial_{r} C_{-} & =0, \\
\partial_{r} h_{-} & =0,
\end{aligned}
$$

which at leading order correspond to the standard outgoing Vaidya metric

$$
d s^{2}=-\left(1-C_{-}\right) d u_{-}^{2}-2 d u_{-} d r+r^{2} d \Omega,
$$

with $C_{-}^{\prime}<0$. Similarly, the case of positive energy density and positive $\tau_{t}^{r}$ is naturally described using advanced null coordinates, resulting in the standard ingoing Vaidya metric

$$
d s^{2}=-\left(1-C_{+}\right) d u_{+}^{2}+2 d u_{+} d r+r^{2} d \Omega,
$$

with $C_{+}^{\prime}>0$.

Equation (4), which relates the differential of $t$ to the differentials of $u_{\mp}$ and $r$, becomes

$$
\begin{aligned}
& d t=e^{-\psi_{\mp}\left(u_{\mp}, r\right)}\left(d u_{\mp} \pm f^{-1} d r\right), \\
& f=1-\frac{C_{\mp}\left(t\left(u_{\mp}, r\right), r\right)}{r}=1-\frac{C_{\mp}\left(u_{\mp}\right)}{r},
\end{aligned}
$$

where the partial differential equation (PDE) for the integrating factor $h=\psi_{\mp}\left(u_{\mp}, r\right)$ is

$$
\partial_{r} \psi_{\mp} \mp f^{-1} \partial_{u_{\mp}} \psi_{\mp}= \pm f^{-2} \partial_{u_{\mp}} f .
$$

In both cases we obtain complex-valued solutions for the transformation from $\left(u_{ \pm}, r\right)$ to $(t, r)$ coordinates. This is a manifestation of the fact that if the NEC is satisfied, there is no finite-time solution that has the Schwarzschild radius $r_{g}(t)$. First we recall some general properties of a first order PDE.

Using the standard procedure for solving a first order linear PDE [35, 36], we set the characteristic system of ordinary differential equations

$$
\begin{aligned}
& \frac{d r}{a}=\frac{d u_{\mp}}{b}=\frac{d \psi_{\mp}}{c}, \\
& a=1, b=\mp f^{-1}, c= \pm f^{-2} \partial_{u_{\mp}} f=\mp f^{-2} C_{\mp}^{\prime} / r .
\end{aligned}
$$

When it is unambiguous we use $\psi=\psi_{\mp}\left(u_{\mp}, r\right), z=u_{ \pm}$, and $C=C_{\mp}$ to simplify the notation in what follows. Two 
independent equations can be selected by choosing, e.g., $z$ as the independent variable. Then the characteristic curves are given by $r=r\left(z, K_{1}\right)$ and $\psi=\psi\left(z, K_{1}, K_{2}\right)$, where $K_{1}$ and $K_{2}$ are constants. The general solution is then implicitly given by

$$
F\left(K_{2}\left(z, K_{1}(z, r), \psi\right)\right)=0
$$

where $F(y)$ is an arbitrary function of a single argument and $K_{1}, K_{2}$ are expressed as functions of $z, r$ and $\psi$. Since we are interested in $\psi$ solely for its capacity as an integrating factor, we take the simplest form of $\psi$ that is compatible with Eq. (C10).

We select two independent equations and use the coordinate $x=r-C(z)$ to obtain the system

$$
\begin{aligned}
& \frac{d r}{d z}=\frac{d C}{d z}+\frac{d x}{d z}=\mp\left(1-\frac{C}{C+x}\right)=\mp \frac{x}{C+x} \\
& \frac{d \psi}{d z}=\frac{1}{f r} \frac{d C}{d z}=\frac{C^{\prime}}{x}
\end{aligned}
$$

where the upper sign corresponds to $z=u_{-}$. To illustrate the key points it is enough to consider early stages of the evolution after formation of the Schwarzschild sphere. In both cases the mass is a linear function of $z$,

$$
C_{ \pm}\left(u_{ \pm}\right)=C_{0}\left(1 \pm \beta u_{ \pm}\right)
$$

where $\beta>0$ is a constant and $C_{0}$ is the $\mathrm{BH}$ mass on the appearance of the Schwarzschild sphere. The evaporating case is described in $\left(u_{-}, r\right)$ coordinates, and the case of growing $\mathrm{BH}$ mass is described in $\left(u_{+}, r\right)$ coordinates. We also consider only regions that are close to the Schwarzschild radius, $x \ll C$. The terms $\beta z C_{0}$ and $x$ are of the same order of magnitude, and we can keep only the first order expression when expanding the denominator of Eq. (C11).

Consider first the outgoing Vaidya metric. Solving Eq. (C11) we obtain (in the leading order)

$$
K_{1}=u_{-}+C_{0} \ln \left(\frac{x}{C_{0}}-\beta C_{0}\right) .
$$

Substituting $x\left(u_{-}\right)$into Eq. (C12) and integrating, we find

$$
K_{2}=\psi_{-}+\ln \left(e^{K_{1} / C_{0}}+\beta C_{0} e^{u_{-} / C_{0}}\right)
$$

For the ingoing Vaidya metric Eq. (C11) gives

$$
K_{1}=u_{+}-C_{0} \ln \left(\frac{x}{C_{0}}-\beta C_{0}\right)
$$

and

$$
K_{2}=\psi_{+}-u_{+} / C_{0}+\ln \left(\beta C_{0}+e^{\left(u_{+}-K_{1}\right) / C_{0}}\right) .
$$

We see that $\psi_{ \pm}\left(u_{ \pm}\right)$must be complex valued as expected for complex $C$ and $h$ in the case of $\Xi=\Upsilon^{2}$. On the other hand, the case of $\Xi=-\Upsilon^{2}$ and positive incoming flux results in a real-valued transformation that connects two coordinate representations of the same physical situation.
[1] V. P. Frolov and I. D. Novikov, Black Holes: Basic Concepts and New Developments (Kluwer, Dordrecht, 1998).

[2] C. Bambi, Black Holes: A Laboratory for Testing Strong Gravity (Springer, Singapore, 2017); Astrophysical Black Holes, edited by F. Haardt, V. Gorini, U. Moschella, A. Trever, and M. Colpi (Springer, Heidelberg, 2016).

[3] V. Cardoso and P. Pani, Nat. Astron. 1, 586 (2017).

[4] M. Visser, Proc. Sci. BHs, GRandStrings2008 (2008) 001; Phys. Rev. D 90, 127502 (2014).

[5] S. W. Hawking and G. F. R. Ellis, The Large Scale Structure of Space-Time (Cambridge University Press, Cambridge, England, 1973).

[6] N. D. Birrel and P. C. W. Davies, Quantum Fields in Curved Space (Cambridge University Press, Cambridge, England, 1986).

[7] R. Brout, S. Massar, R. Parentani, and P. Spindel, Phys. Rep. 260, 329 (1995).

[8] R. B. Mann, Black Holes: Thermodynamics, Information, and Firewalls (Springer, New York, 2015).

[9] A. Ashtekar and M. Bojowald, Classical Quantum Gravity 22, 3349 (2005).

[10] S. A. Hayward, Phys. Rev. Lett. 96, 031103 (2006).

[11] C. J. Fewster and G. J. Galloway, Classical Quantum Gravity. 28, 125009 (2011); P. J. Brown, C. J. Fewster, and E.-A. Kontou, Gen. Relativ. Gravit. 50, 121 (2018).

[12] P. Chen, Y. C. Ong, and D.-h. Yeom, Phys. Rep. 603, 1 (2015).
[13] C. Barceló, S. Liberati, S. Sonego, and M. Visser, J. High Energy Phys. 02, 003 (2011).

[14] V. Faraoni, Galaxies 1, 114 (2013).

[15] B. Krishnam, Quasi-local horizons, in Springer Handbook of Spacetime, edited by A. Ashtekar and V. Petkov (Springer, New York, 2014), p. 527.

[16] S. W. Hawking, Nature (London) 248, 30 (1974).

[17] A. Paranjape and T. Padmanabhan, Phys. Rev. D 80, 044011 (2009).

[18] V. Baccetti, R. B. Mann, and D. R. Terno, Classical Quantum Gravity 35, 185005 (2018).

[19] J. M. Bardeen, Phys. Rev. Lett. 46, 382 (1981).

[20] M. Blau, Lecture Notes on General Relativity.

[21] V. Mukhanov, Physial Foundations of Cosmology (Cambridge University Press, 2005), Sec. 2.3.

[22] G. C. McVittie, Mon. Not. R. Astron. Soc. 93, 325 (1933); N. Kaloper, M. Kleban, and D. Martin, Phys. Rev. D 81, 104044 (2010); A. M. da Silva, M. Fontanini, and D. C. Guariento, Phys. Rev. D 87, 064030 (2013).

[23] J. M. Bardeen, arXiv:1406.4098; P. Binétruy, A. Helou, and F. Lamy, Phys. Rev. D 98, 064058 (2018).

[24] A. Levi and A. Ori, Phys. Rev. Lett. 117, 231101 (2016).

[25] R. M. Corless, G. H. Gonnet, D. E. G. Hare, D. J. Jeffrey, and D. E. Knuth, Adv. Comp. Math. 5, 329 (1996).

[26] P. Martín-Moruno and M. Visser, Classical and Semi-classical Energy Conditions, in Wormholes, Warp Drives and Energy Conditions, edited by F. N. S. Lobo (Springer, New York, 
2017), p. 193.

[27] C. J. Fewster, Quantum Energy Conditions, in Wormholes, Warp Drives and Energy Conditions, edited by F. N. S. Lobo (Springer, New York, 2017), p. 215.

[28] L. H. Ford, A. D. Helfer, and T. A. Roman, Phys. Rev. D 66, 124012 (2002).

[29] C. J. Fewster and M. J. Pfenning, J. Math. Phys. 47, 082303 (2006).

[30] T. Padmanabhan, Gravity and Spacetime: AN Emergent Perspective, in Springer Handbook of Spacetime, edited by A. Ashtekar and V. Petkov (Springer, New York, 2014), p. 213.

[31] V. Baccetti, S. Murk, and D. R. Terno, arXiv:1812.07727.
[32] V. Baccetti, V. Hussain, and D. R. Terno, Entropy 19, 17 (2017).

[33] K. Maruyama, F. Nori, and V. Vedral, Rev. Mod. Phys. 81, 1 (2009); M. B. Plenio and V. Vitelli, Cont. Phys. 42, 1 (2001).

[34] M. E. Carrington, G. Kunstatter, J. Louko, and L. J. Zhou, Phys. Rev. D 98, 024035 (2018).

[35] R. Courant and D. Hilbert, Methods of Modern Mathematical Physics (Wiley Interscience, New York, 1961), Vol. 2.

[36] L. C. Evans, Partial Differential Equations (AMS, Providence, 1998). 